\title{
Solubilization of poorly soluble photosensitizer hypericin by polymeric micelles and polyethylene glycol *
}

\author{
Diana Búzová1, Peter Kasák ${ }^{2}$, Pavol Miškovský1,3 and Daniel Jancura ${ }^{1}$ \\ ${ }^{1}$ Department of Biophysics, P. J. Safarik University, Kosice, Slovak Republic \\ ${ }^{2}$ Polymer Institute of the Slovak Academy of Sciences, Bratislava, Slovak Republic \\ ${ }^{3}$ International Laser Center, Bratislava, Slovak Republic
}

\begin{abstract}
Hypericin (Hyp) is a promising photosensitizer for photodiagnosis and photodynamic therapy of cancer. However, Hyp has a large conjugated system and in aqueous solutions forms insoluble aggregates which do not possess biological activity. This makes intravenous injection of Hyp problematic and restricts its medical applications. To overcome this problem, Hyp is incorporated into drug delivery systems which can increase its solubility and bioavailability. One of the possibilities is utilization of polymeric micelles. The most used hydrophilic block for preparation of polymeric micelles is polyethylen glycol (PEG). PEG is a polymer which for its lack of immunogenicity, antigenicity and toxicity obtained approval for use in human medicine. In this work we have studied the solubilization of Hyp aggregates in the presence of PEG-PE and PEG-cholesterol micelles. The concentration of polymeric micelles which allows total monomerization of Hyp corresponds to the critical micellar concentration of these micelles $\left(\sim 10^{-6} \mathrm{M}\right)$. We have also investigated the effect of the molecular weight and concentration of PEG on the transition of aggregated Hyp to its monomeric form. PEGs with low molecular weight $(<1000 \mathrm{~g} / \mathrm{mol})$ do not significantly contribute to the solubilization of Hyp. However, PEGs with molecular weight $>2000 \mathrm{~g} / \mathrm{mol}$ efficiently transform Hyp aggregates to the monomeric state of this photosensitizer.
\end{abstract}

Key words: Hypericin - Photodynamic therapy - Polyethylene glycol - Polymeric micelles - Solubilization

\section{Introduction}

Hypericin (Hyp), 7,14-dione-1,3,4,6,8,13-hexahydroxy 10,11-dimethyl-phenanthrol [1,10,9,8-opqra] perylene (Fig. 1), is a natural photosensitizer occuring in plants of the genus Hypericum. Hyp for its physico-chemical properties is a very promising agent in photodiagnosis and photodynamic therapy (PDT) of cancer. Under light illumination Hyp displays antiproliferative and cytotoxic effects on many tumor cell lines. This property, together with minimal dark toxicity, certain tumor selectivity and high clearance rate from the host body, enables this compound to belong between the

Corresponding author: Daniel Jancura, Department of Biophysics, P. J. Safarik University, Jesenna 5, 04154 Kosice, Slovak Republic E-mail: jancura@upjs.sk

* This article was presented at the $5^{\text {th }}$ Slovak Biophysical Symposium, organised by The Slovak Biophysical Society in Bratislava, March 19-21, 2012 most effective natural photosensitizers (see reviews Miskovsky 2002; Kiesslich et al. 2006; Kober et al. 2008; Kramer and Verwanger 2012).

Hyp is a hydrophobic molecule and is readily soluble in polar organic solvents like dimethyl sulfoxide (DMSO), acetone, ethyl acetate and ethanol. This compound is sparingly soluble in non-polar organic solvents and oil, and in aqueous environment forms insoluble non-fluorescent aggregates (Falk and Meyer 1994; Falk 1999). The formation of aggregates significantly suppresses Hyp photodynamic activity (Falk and Meyer 1994; Kascakova et al. 2005; van de Putte et al. 2006). Moreover, this aqueous insolubility makes intravenous injection of Hyp problematic and restricts its medical applications. Administration of aggregated Hyp strongly hinders a selective accumulation in tumor tissues and a pronounced uptake in liver, spleen and lung is observed (van de Putte et al. 2006). To overcome this problem, Hyp is usually incorporated into various drug delivery systems, which can increase its solubility and bioavailability. 
<smiles></smiles>

Figure 1. Structure of hypericin.

There are many attempts to find suitable transport systems for Hyp (and other hydrophobic drugs). Up to now, several delivery systems have been utilized for Hyp transport: Hyploaded polymeric nanoparticles of polylactic acid (ZeisserLabouebe et al. 2006; Zeisser-Labouebe et al. 2009), Hyp bound to polyvinyl-pyrrolidone (Kubin et al. 2008) and N-methyl-pyrrolidone (Saw et al. 2005, 2006), Hyp embedded in liposomes (Angelini et al. 1997; Roslaniec et al. 2000; Galanou et al. 2008), Hyp in transferin conjugated polyethylen glycol (PEG)-liposomes (Derycke and de Witte 2002) and DMSO-PEG solutions (Huygens et al. 2005). The transport of Hyp in a complex with delivery system usually results in<smiles>CCCOCCCO</smiles>

PEG

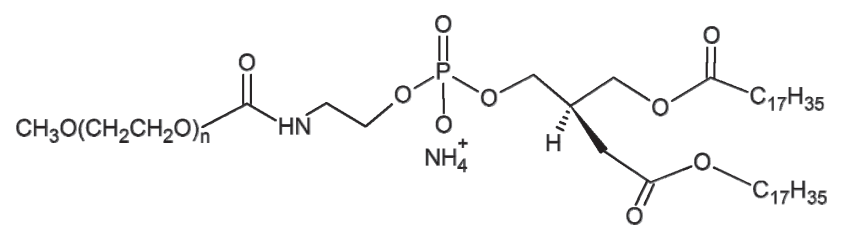

PEG-PE

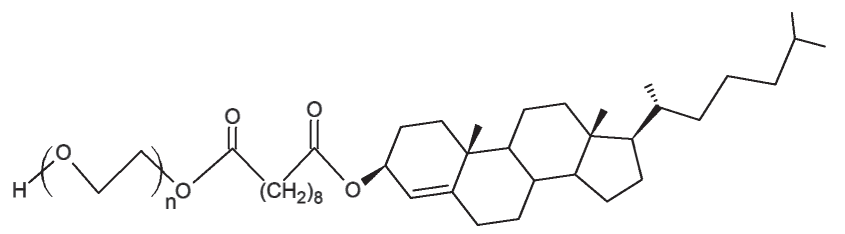

PEG-Chol

Figure 2. Structure of PEG, PEG-PE and PEG-chol. improved tumor to normal tissues ratio and a relatively fast elimination of Hyp from a biological organism (van de Putte et al. 2006). However, the nature of the vehicle was found to be of critical importance to the tissue distribution of Hyp as it determines the physico-chemical interactions of Hyp and its transfer to plasma lipoproteins (van de Putte et al. 2006). The study of Hyp distribution in human plasma has shown that Hyp molecules are predominantly associated with low-density lipoproteins (LDL) and only relatively small amounts are bound to high-density lipoproteins (HDL) and proteins (mainly serum albumin) (Chen et al. 2001). In the presence of the plasma (lipo)proteins Hyp aggregates can redissociate resulting in fluorescent monomeric and biological active form of this molecule (Falk and Meyer 1994; Lavie et al. 1995; Huygens et al. 2005; Kascakova et al. 2005). Our group has published several articles about the properties of Hyp/LDL complexes (Kascakova et al. 2005; Mukherjee et al. 2008; Gbur et al. 2009; Huntosova et al. 2010; Buriankova et al. 2011).

In our attempt to evaluate more deeply the solubilization process of Hyp in aqueous solutions in the presence of different delivery systems, we present data on the incorporation of Hyp into polymeric micelles from PEGylated phospholipids, PEG-phosphatidyl ethanolamine (PEG-PE), and PEGylated cholesterol (PEG-chol), and the effect of the molecular weight and concentration of PEG on the transition of aggregated Hyp to its monomeric form (Fig. 2).

Polymeric micelles are very promising drug carrier systems which received growing scientific attention in the recent years (van Nostrum 2004; Sezgin et al. 2007; Torchilin 2007). The advantageous properties of polymeric micelles such as the ability of incorporating and protecting poorly soluble drugs, improving drugs bioavailability, targeting ability, long circulation in humam plasma and easy production, make this delivery system very attractive for practical medical applications (Andresen et al. 2005; Lukyanov and Torchilin 2004; Torchilin 2007). A significant step in the development of long circulating polymeric micelles came with inclusion of PEG to liposome composition. The presence of PEG on the surface of the liposomal carrier has been shown to extend blood circulation time, while reducing mononuclear phagocyte system uptake (stealth liposomes) (Veronese and Pasut 2005; van Vierken et al. 2007). This technology has resulted in a large number of liposome formulations encapsulating active molecules with high target efficiency and activity (Hamblin et al. 2003; Roby et al. 2006; Sezgin et al. 2007). Further, by synthetic modification of the terminal PEG molecule, stealth liposomes can be actively targeted to tumor cells by monoclonal antibodies or tumor specific ligands (Immordino et al. 2006).

PEG is the most popular hydrophilic polymer for the surface modification of drug carriers, or for the composing the corona of therapeutic micelles. It has proven to be an 
efficient steric protector of various biologically active molecules and particular drug delivery systems. This polymer is enexpenisve, has low toxicity, the lack of immunogenicity, antigenicity and toxicity, high solubility in water and organic solvents, high flexibility of the chain and has been approved for applications by drug regulatory agencies (Lukyanov and Torchilin 2004; Fishburn 2008; Wattendorf and Hans 2008; Bailon and Won 2009).

In this work we have studied the solubilization of Hyp aggregates in the presence of PEG-PE and PEG-chol micelles, as well as PEG alone, with the aim to provide quantitative characterization of the transition of aggregated form of Hyp to its monomeric, biologically active state in aqueous solutions. The presented results demonstrate the applicability of the polymeric micelles and PEG as suitable transport systems for highly hydrophobic Hyp. These delivery vehicles significantly improve aqueous solubility of Hyp, which enhance possibility of effective and selective delivery of this drug to tumor tissue during PDT.

\section{Materials and Methods}

\section{Chemicals}

Hyp, DMSO, phosphate buffer (PBS), PEG of different molecular weights $(300,600,1000,2000,4600,8000 \mathrm{~g} / \mathrm{mol})$ and $\mathrm{PEG}_{600}$ mono(cholesteryl)ether sebacate (PEG-chol) were obtained from Sigma-Aldrich (Germany), PEGylated phospholipids, $\mathrm{PEG}_{1000}-\mathrm{PE}$ and $\mathrm{PEG}_{5000}-\mathrm{PE}$, were purchased from Avanti Lipids (USA).

\section{Preparation of PEG-PE and PEG-chol micelles}

The stock solutions of PEG-PE and PEG-chol $\left(10^{-2} \mathrm{M}\right)$ were prepared in 100\% DMSO. An aliquot amount of stock solutions was added to PBS ( $\mathrm{pH} 7.4$ ) to achieve the PEGPE (PEG- chol) concentrations between $10^{-8}-10^{-3} \mathrm{M}$. The obtained solutions were then stirred for $10 \mathrm{~min}$ at the temperature $20^{\circ} \mathrm{C}$. The formed self-assembled structures were further characterized by dynamic light scattering (DLS).

\section{Preparation and characterization of Hyp/PEG-PE (Hyp/} PEG-chol) and Hyp/PEG complexes

The stock solution of Hyp $\left(2 \times 10^{-3} \mathrm{M}\right)$ was prepared by dissolving of solid Hyp in $100 \%$ DMSO. The stock solutions of PEG were prepared by dissolving of solid PEG in PBS ( $\mathrm{pH}$ 7.4) and stock solutions of PEG-PE and PEG-chol were prepared by dissolving of the polymers in DMSO. The solutions for experiments were obtained by dilution of the stock solutions of PEG (PEG-PE and PEG-chol) and Hyp with PBS ( $\mathrm{pH} 7.4$ ) and were stored in the dark for $2 \mathrm{~h}$ before measurements. The concentration of Hyp was kept constant $\left(10^{-8}\right.$ and $\left.2 \times 10^{-6} \mathrm{M}\right)$, the concentrations of PEG varied between $10^{-8}-10^{-1} \mathrm{M}$ and for PEG-PE and PEG-chol the concentrations were from the interval $10^{-8}-10^{-4} \mathrm{M}$. The final quantity of DMSO in all experiments was less than $1 \%$. Dialysis using a permeable membrane against PBS solution at $\mathrm{pH} 7.4$ for $18 \mathrm{~h}$ at room temperature was applied to ensure removal of non-incorporated Hyp molecules.

\section{Dynamic light scattering}

DLS measurements for size characterizations of PEG-PE and PEG-chol particles were performed by Malvern Instruments Zetasizer Nano-ZS instrument (Malvern, Worcs. UK) equipped with a $4 \mathrm{~mW} \mathrm{He}-\mathrm{Ne}$ laser $(633 \mathrm{~nm})$ operating at $90^{\circ}$ angle and at temperature of $20^{\circ} \mathrm{C}$.

\section{UV/VIS absorption and fluorescence measurements}

UV-VIS absorption spectra were measured on Shimadzu (UV-2401 PC) UV-VIS spectrophotometer in 200-700 nm range. Fluorescence emission spectra were obtained using Shimadzu RF-5301 PC fluorimeter (Kyoto, Japan) in Quartz SUPRASIL cuvette $(10 \mathrm{~mm}$ ) from Heraus Quarzglas $\mathrm{GmbH}$. The excitation line was $515 \mathrm{~nm}$ and the intensity of fluorescence of Hyp was recorded in 560-700 nm region. Data were analyzed by the program Origin 8.0.

\section{Results and Discussion}

\section{Characterization of PEG-PE and PEG-chol micelles}

The hydrodynamic diameters of PEG-PE and PEG-chol micelles were determined by DLS method (Fig. 3). The

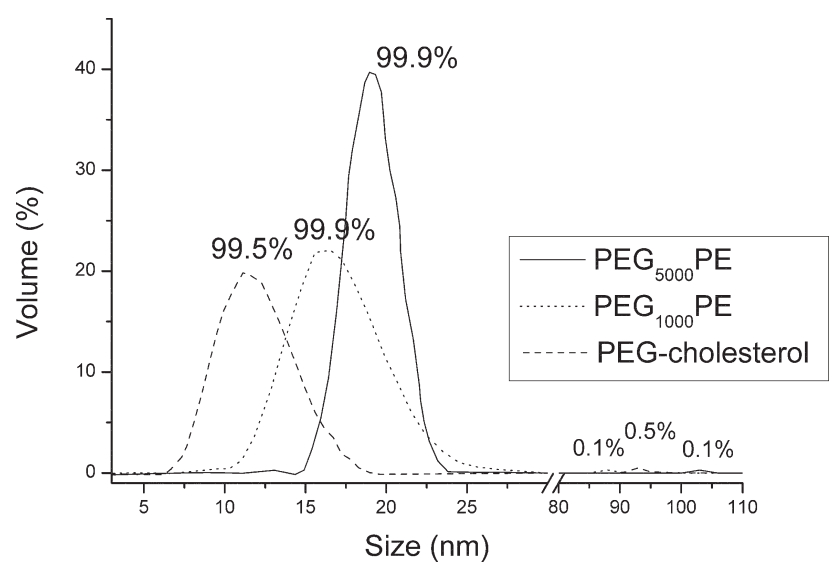

Figure 3. Size distribution of $\mathrm{PEG}_{1000}-\mathrm{PE}\left(10^{-5} \mathrm{M}\right), \mathrm{PEG}_{5000}-\mathrm{PE}$ $\left(10^{-5} \mathrm{M}\right)$ and PEG-chol $\left(10^{-5} \mathrm{M}\right)$ polymeric micelles determined by dynamic light scattering. 
obtained results show that the mean diameters for prepared PEG-PE and PEG-chol micelles are within the characteristic micelle size reported for these systems (Sezgin et al. 2006): 13-18 $\mathrm{nm}$ for $\mathrm{PEG}_{5000}-\mathrm{PE}, 10-15 \mathrm{~nm}$ for $\mathrm{PEG}_{1000}-\mathrm{PE}$, and 8-15 nm for PEG-chol, depending on the concentration of PEG-PE or PEG-chol. As it was previously proposed, this relatively small size of PEG-PE and PEG-chol micelles would be beneficial for oral drug delivery (Sezgin et al. 2007). The stability of the micelles were followed for 5 days at $20^{\circ} \mathrm{C}$ and no changes in the micelle size or size distribution were observed during this time period (not shown). This finding is in good accordance with the previous work of Roby and co-workers, where the stability of the PEG polymeric micelles was investigated (Roby et al. 2006).

\section{Solubilization of Hyp by PEG-PE and PEG-chol micelles}

The fluorescence emission spectra of Hyp $\left(10^{-8} \mathrm{M}\right)$ in different solvents are presented in Fig. 4. Hyp exhibits no fluorescence in aqueous PBS. This fact is well known and is attributed to the formation of non-fluorescent Hyp aggregates in aqueous solutions (Falk and Meyer 1994; Kascakova et al. 2005). The fluorescence spectra of monomeric Hyp are represented by the spectra of Hyp dissolved in DMSO and in the complex with LDL (Hyp/LDL = 10:1) (Kascakova et al. 2005). The solubilization activity of PEG-PE is manifested by the fact, that the fluorescence intensity of Hyp in the presence of PEG-PE is almost identical to those intensities observed in DMSO and LDL, where Hyp is readily soluble (Fig. 4). It means that the quantum yield of the fluorescence of Hyp incorporated in PEG-PE is similar to those for Hyp

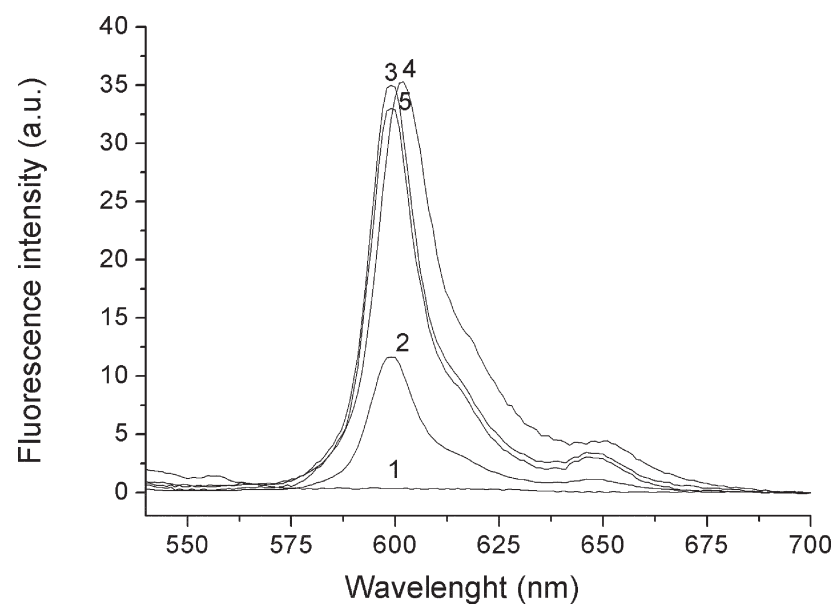

Figure 4. Fluorescence emission spectra (excitation wavelenght was at $515 \mathrm{~nm})$ of hypericin $\left(10^{-8} \mathrm{M}\right)$ in different solvent conditions: in PBS ( $\mathrm{pH}=7.4$ ) aqueous solution (1); in the presence of $\mathrm{PEG}_{8000}$ $\left(10^{-3} \mathrm{M}\right)(2)$; in the presence of LDL $\left(10^{-9} \mathrm{M}\right)(3)$; in the presence of PEG $\mathrm{P}_{500}-\mathrm{PE}\left(10^{-4} \mathrm{M}\right)(4)$; and in $100 \%$ DMSO (5). in LDL or dissolved in various polar organic solvents (e.g. DMSO), where the quantum yield of Hyp fluorescence has been determined to be 0.2 (Falk and Meyer 1994; Hadjur et al. 1996; Kascakova et al. 2005).

Using the Hyp characteristic that only the monomeric form of this compound is fluorescent, we have investigated the transition of Hyp from aggregated to monomeric state. The incorporation of Hyp to PEG-PE and PEG-chol micelles (using PEGs with two different molecular weights: $1000 \mathrm{~g} / \mathrm{mol}, 5000 \mathrm{~g} / \mathrm{mol}$ ) was studied under the excitation $515 \mathrm{~nm}$ and the emission spectra were recorded in the 525-700 nm region. The intensity of Hyp fluorescence at its maximum (599 $\mathrm{nm}$ ) as a function of PEG-PE (PEGchol) concentration is presented in Fig. 5. The fluorescence intensity substantially increases with the increase of PEG-PE (PEG-chol) concentration and at the values, $2.8 \times$ $10^{-6} \mathrm{M}$ for $\mathrm{PEG}_{1000^{-}} \mathrm{PE}, 1.6 \times 10^{-6} \mathrm{M}$ for $\mathrm{PEG}_{5000^{-}} \mathrm{PE}$ and $1.9 \times 10^{-6} \mathrm{M}$ for PEG-chol, the intensity reaches the half of its maximum. It means that at those concentrations $\left(\mathrm{EC}_{50}\right)$, $50 \%$ of Hyp molecules occur in the monomeric state. To confirm this finding, UV-VIS absorption experiments have been realized. It is known that UV-VIS absorption spectra of aggregated and monomeric Hyp are different and this is reflected by the ratio of the absorbance at $554 \mathrm{~nm}$ and $597 \mathrm{~nm}, \mathrm{~A}_{554} / \mathrm{A}_{597}$ (Fig. 6A). For the fully aggregated Hyp (spectrum in aqueous $\mathrm{PBS}$ ) this ratio is $\sim 1.2$, for the monomeric form this value reaches $\sim 0.5$ (spectrum of Hyp in DMSO or at high PEG-PE concentrations) (Kascakova et al. 2005). We used these absorption spectra to construct the dependence of $\mathrm{A}_{554} / \mathrm{A}_{597}$ on the population of the monomeric form of Hyp (graph inserted in Fig. 6A). Us-

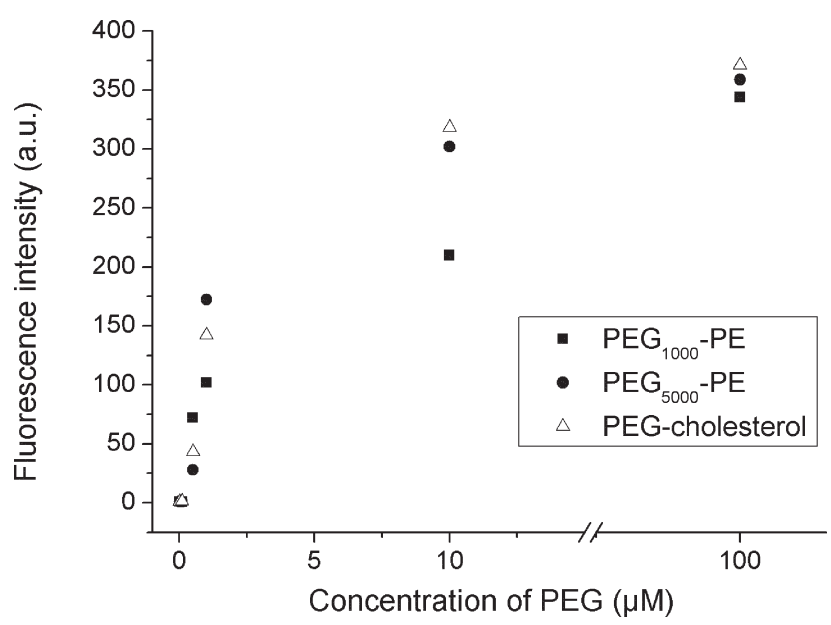

Figure 5. Dependence of the fluorescence intensity of hypericin (2 $\left.\times 10^{-6} \mathrm{M}\right)$ at its maximum $(599 \mathrm{~nm})$ on $\mathrm{PEG}_{1000}-\mathrm{PE}, \mathrm{PEG}_{5000}-\mathrm{PE}$ and PEG-chol concentration in PBS aqueous solution ( $\mathrm{pH}$ 7.4). Excitation line was $515 \mathrm{~nm}$. 
ing this reference curve, the quantification of population of Hyp monomers at different PEG-PE (PEG-chol) concentrations was then determined (Fig. 6B). The obtained curves are in good correlation with those obtained from fluorescence experiments and confirm that PEG-PE and PEG-chol micelles can fully solubilized aggregates of Hyp in aqueous environment.

The $\mathrm{EC}_{50}$ values for PEG-PE (PEG-chol) approximately correspond to the critical micellar concentrations (CMC) of PEG-PE conjugates $\left(\sim 10^{-6}-10^{-5} \mathrm{M}\right)$ (Lukyanov and Torchilin 2004). It signifies that after formation of polymeric micelles, molecules of Hyp are incorporated into the micelles and are transformed from the aggregated to the monomeric state. This is in accordance with the previous findings of Derycke and de Witte (2002). These authors estimated that one PEG-PE liposome (diameter $146 \mathrm{~nm}$ ) can contain 20000 molecules of Hyp. In our case, the diameters of PEG-PE micelles are around 10 $\mathrm{nm}$. If we utilize simple model for the quantification of phospholipids molecules in one PEG-PE micelle (100 nm liposomes is built up of 100000 phospholipids (Derycke and de Witte 2002)), then $10 \mathrm{~nm}$ micelle is built up of $\sim 500$ phospholipids. From this simple assumption we can estimate that in the case when concentration of PEG-PE is $10^{-6} \mathrm{M}$ (which corresponds to $\sim 2 \times 10^{-9} \mathrm{M}$ concentration of PEG-PE micelles), the average number of Hyp molecule in one micelle particle is $\sim 1000$, for Hyp with concentration of $2 \times 10^{-6} \mathrm{M}$, and only 5 molecules in the case of Hyp concentration $10^{-8} \mathrm{M}$.

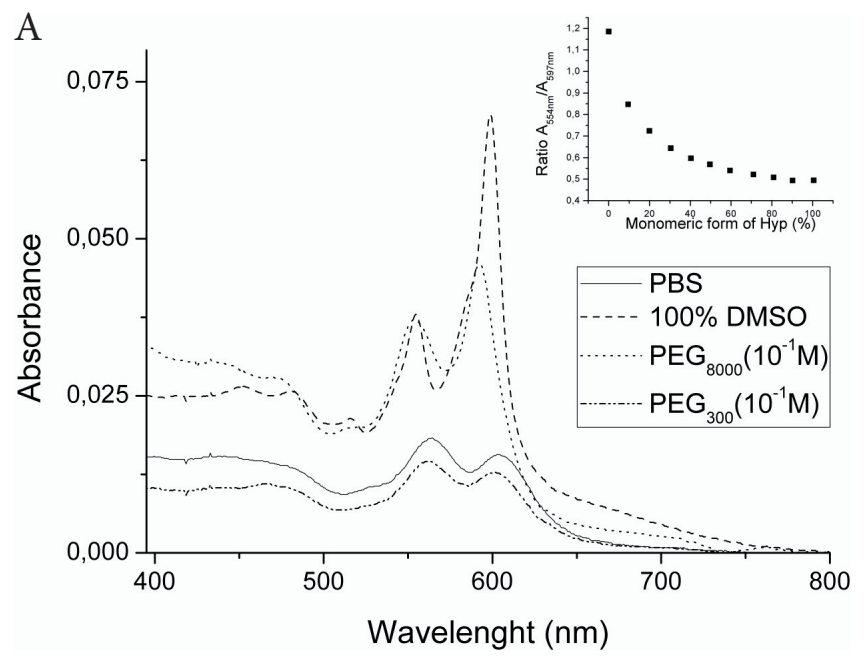

With respect to the stability of Hyp complex with PEGmicelles (liposomes), Derycke and de Witte showed that in the case when complex Hyp-PEG-liposome was incubated in medium containing fetal calf serum, the substantial amount of Hyp is lost from the liposomes (Derycke and de Witte 2002). The reason is that Hyp exhibits a high affinity for lipoproteins and albumin presented in the serum. They suggested several ways to solve this problem, e.g. using multilamellar liposomes or incorporation of water soluble derivates of Hyp. We propose that a lowering of the concentration of Hyp in one polymeric micelle (liposome) particle should be another way for the higher stability of the complex. It seems that 20000 Hyp molecules in one PEG-liposome is too high and transfer of Hyp from such complex to serum lipoproteins is very probable. Incorporation of „only“ tens or hundreds Hyp molecules into PEG-micelles, as it is realized in this work when the concentration of incorporated Hyp is low $\left(10^{-8} \mathrm{M}\right)$, could substantially decrease the percentage of Hyp transfer from polymeric micelles to serum (lipo)proteins. However, this suggestion needs to be experimentally confirmed.

In conclusion of this part it is necessary to mention that relatively very low CMC values for PEG-PE and PEG-cholesterol micelles indicate that these micelles will maintain their integrity even upon strong dilution (for example in the blood during a therapeutic application) and their application as drug delivery system of highly water insoluble drug, like Hyp, is very promising and deserve further studies.

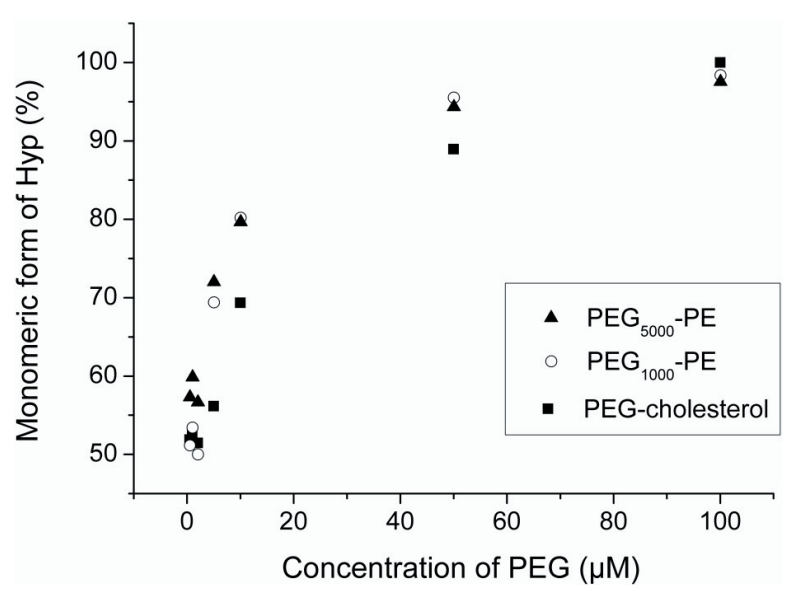

Figure 6. A. Absorption spectra of hypericin $\left(2 \times 10^{-6} \mathrm{M}\right)$ in the different solvent conditions: PBS (pH 7.4); $100 \%$ DMSO; the presence of PEG $8000\left(10^{-1} \mathrm{M}\right)$, and the presence of $\mathrm{PEG}_{300}\left(10^{-1} \mathrm{M}\right)$. Inserted graph presents the dependence of the ratio of intensities of the main absorbtion bands of hypericin on the population of the monomeric form of hypericin. This dependence is based on the spectral mixing of absorption spectra of hypericin in PBS (hypericin fully aggregated) and in LDL at Hyp/LDL molar ratio of 30:1 (monomeric hypericin) (Kascakova et al. 2005). B. Population of hypericin monomers in $\mathrm{PEG}_{1000}-\mathrm{PE}, \mathrm{PEG}_{5000}-\mathrm{PE}$ and PEG-Chol micelles as a function of the micelles concentration. 


\section{Monomerization of Hyp aggregates in the presence of PEG}

The fluorescence intensity of Hyp as a function of PEG concentration for polymers with different molecular weights $(300,600,1000,2000,4600,8000 \mathrm{~g} / \mathrm{mol})$ is presented in Fig. 7 (Hyp concentration is $2 \times 10^{-6} \mathrm{M}$ ). The fluorescence increases with the rise of PEG concentration which means that PEG alone enables transformation of Hyp from aggregated to its monomeric form. It is observed that PEGs with molecular weight under $1000 \mathrm{~g} / \mathrm{mol}$ have only slight influence on the monomerization of Hyp aggregates. However, PEGs with high molecular weight $(>2000 \mathrm{~g} / \mathrm{mol})$ enable aqueous solubilizition of Hyp and can partially dissociate Hyp aggregates into monomeric state. This effect is possible due to the structure of PEG (Fig. 2), which makes PEG miscible with water trough hydrogen bonding. The hydrophobic hydrocarbon region helps to break the hydrogen bonding between water molecules, thus reducing overall intermolecular interactions, so PEG may assist to reduce the dipole moment of water and allow hydrophobic compounds to fit in (Millard et al. 2002; Nandi et al. 2003).

Similarly as in the case of Hyp incorporation into PEG-PE (PEG-cholesterol) micelles, we have measured dependence of absorption intensity of Hyp on PEG concentration for PEGs with different molecular weights $(300,600,1000,2000$, $4600,8000 \mathrm{~g} / \mathrm{mol}$ ). The absorption spectra of Hyp for $\mathrm{PEG}_{300}$ and $\mathrm{PEG}_{8000}$ are shown on Fig. 6A. For PEGs with lower molecular weight $(<1000 \mathrm{~g} / \mathrm{mol})$, the absorption spectrum of Hyp is similar to the spectrum in PBS and even increasing of PEG concentration does not lead to any significant change of the spectrum, and Hyp remains in its aggregated form. With an increase of PEG molecular weight, the absorption spectrum of Hyp is changing. The spectrum become simi- lar to that one in DMSO, it means that Hyp transits from aggregate to its monomeric form. The position of the Hyp absorption maxima in the visible region are slightly shifted to shorter wavelength with increasing PEG concentration (from $597 \mathrm{~nm}$ to $592 \mathrm{~nm}$ ) (Fig. 6A). This hypsochromic shift is a result of an increase of the polarity of the solvent.

The obtained absorption spectra provide opportunity to quantify the percentage of monomeric form of Hyp in the solution. Using the reference curve (see inserted graph in Fig. 6A), which manifests the dependence of the absorbance ratio $A_{554} / A_{597}$ on the population of Hyp monomeric form, we determined the relative equilibrium between monomeric and aggregated states of this molecule. Fig. 8 presents corresponding data in the presence of $\mathrm{PEG}_{300}, \mathrm{PEG}_{2000}$ and $\mathrm{PEG}_{8000}$. It is evident that even at the highest PEG concentrations we have used $\left(10^{-1} \mathrm{M}\right)$, the molecules of Hyp are not fully monomerized. However, the PEGs with high molecular weight $(>5000 \mathrm{~g} / \mathrm{mol})$ can substantially decrease the aggregation state (in the presence of $10^{-1} \mathrm{M}$ of $\mathrm{PEG}_{8000}, 75 \%$ of Hyp molecules are in the monomeric state (Fig. 8)).

The applicability of PEG for solubilization of Hyp was previously shown by using $\mathrm{PEG}_{400}$ as a solvent. Hyp was completely dissolved in pure $\mathrm{PEG}_{400}$ (Huygens et al. 2005). Moreover, it was found that this system maintained the stability of the compounds for at least 120 days when stored at $4^{\circ} \mathrm{C}$ and $37^{\circ} \mathrm{C}$. Huygens and co-workers suggested that $\mathrm{PEG}_{400}$ is an excellent Hyp formulation and suitable vehicle for the storage of Hyp prior to preparation of the bladder instillation solution. In our experiments we have observed significant solubilization of Hyp only for PEGs with the molecular weight over $2000 \mathrm{~g} / \mathrm{mol}$. However, we have utilized PEGs with maximal concentration $10^{-1} \mathrm{M}$ in solution. The obtained result shows that Hyp can be solubilized not only

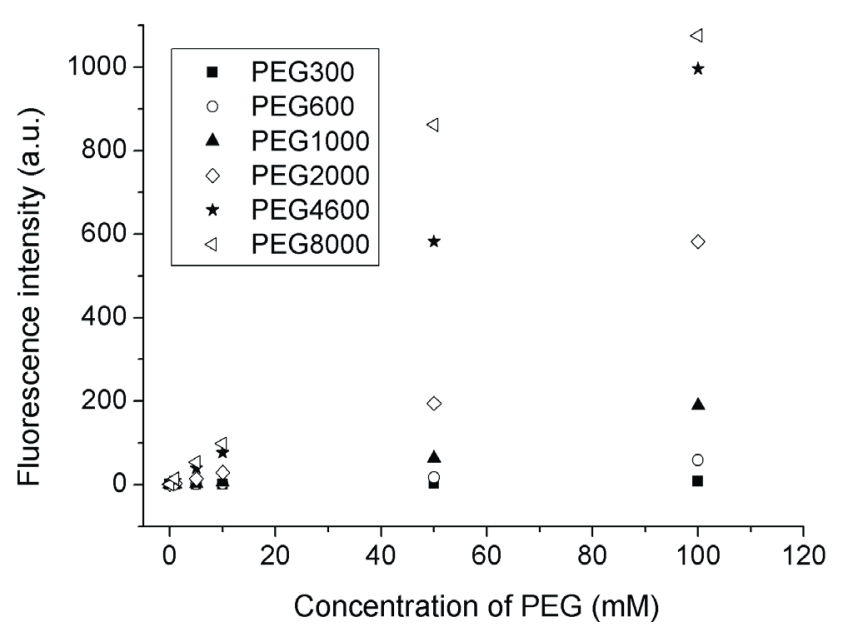

Figure 7. Dependence of the fluorescence intensity of hypericin $(2 \times$ $10^{-6} \mathrm{M}$ ) at its maximum $-599 \mathrm{~nm}$ on PEG concentration for PEGs with different molecular weights. Excitation line was $515 \mathrm{~nm}$.

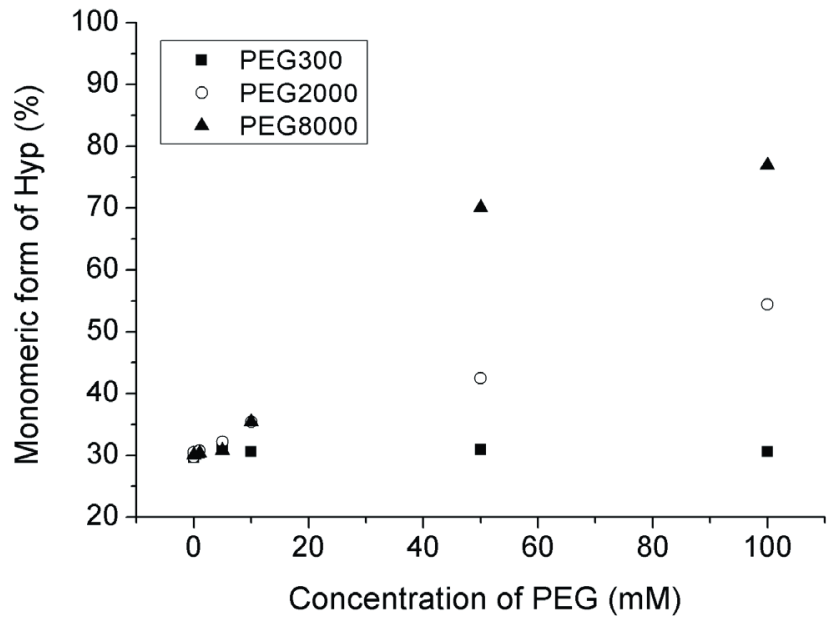

Figure 8. Population of hypericin monomers in the presence of $\mathrm{PEG}_{300}, \mathrm{PEG}_{2000}$ and $\mathrm{PEG}_{8000}$ as a function of the concentration of PEG. 
in pure PEG as it was observed previously (Huygens et al. 2005), but also in PEG aqueous solutions. However, the molecular weight of PEG needs to be higher than $2000 \mathrm{~g} / \mathrm{mol}$. These findings suggest that utilization of PEG is one of the possibilities how to maintain Hyp in its biological active monomeric state also in aqueous environment.

\section{Conclusion}

The present work shows that PEG-PE and PEG-chol micelles can be used for solubilization of poorly soluble photosensitizer Hyp in aqueous solutions. The concentration of PEG-PE (PEG-chol) which allows total monomerization of Hyp corresponds to the CMC of these micelles $\left(\sim 10^{-6} \mathrm{M}\right)$. In accordance with the previous works we concluded that PEG-based micelles can be used as drug delivery system of highly water insoluble drugs, like Hyp, with the perspective of higher bioavailability, selectivity and targeting of the drug.

We have also shown that PEG alone can improve the aqueous solubility of Hyp. It was found that the molecular weight and concentration of PEG polymers affects a transition of Hyp from aggregate form to its monomeric form in aqueous solution. PEGs with low molecular weight $(<1000 \mathrm{~g} / \mathrm{mol})$ do not significantly contribute to the solubilization of Hyp. However, PEGs with molecular weight $>2000 \mathrm{~g} / \mathrm{mol}$ efficiently transform Hyp aggregates to the monomeric state of this photosensitizing agent. These findings suggest that utilization of PEG is one of the possibilities how to mantain Hyp in its biological active monomeric state.

We hope that our characterization of both Hyp-polymeric micelle and Hyp-PEG systems will contribute to the endeavour to find suitable drug delivery system for systemic, effective, and safety Hyp administration into a human body during PDT.

Acknowledgments. This work was supported by the Slovak Research and Development Agency contracts APVV-0242-11 and LPP-0072-07, the Scientific Grant Agency of the Ministry of Education of Slovak Republic (grant VEGA-0164-09), and by the Agency of the Ministry of Education of the Slovak Republic for the Structural funds of the European Union, Operational program Research and Development (contracts: CEVA II (ITMS code: 26220120040) and SEPO II (ITMS code: 26220120039)).

\section{References}

Andresen T. L., Jensen S. S., Jorgensen K. (2005): Advanced strategies in liposomal cancer therapy: Problems and prospects of active and tumor specific drug release. Prog. Lipid Res. 44, 68-97 http://dx.doi.org/10.1016/j.plipres.2004.12.001

Angelini N., Cubeddu R., Lenci F., Losi A., Pifferi A., Sgarbossa A., Taroni, P., Vecli A., Viappiani C. (1997): Artificial models of bio- logical photoreceptors: effect of quenchers on the fluorescence properties of hypericin embedded in liposomes. J. Photochem. Photobiol. B: Biology 38, 245-252 http://dx.doi.org/10.1016/S1011-1344(96)07475-1

Bailon P., Won Ch. Y. (2009): PEG - modified biopharmaceuticals. Expert Opin. Drug Deliv. 6, 1-16 http://dx.doi.org/10.1517/17425240802650568

Buriankova L., Buzova D., Chorvat D. Jr., Dureau F., Brault D., Miskovsky P., Jancura D. (2011): Kinetics of hypericin association with low-density lipoproteins. Photochem. Photobiol. 87, 56-62 http://dx.doi.org/10.1111/j.1751-1097.2010.00847.x

Chen B., Xu Y., Roskans T., Delaey E., Agostinis P., Vandenheede J. R., de Witte P. A. M. (2001): Efficacy of antitumoral photodynamic therapy with hypericin: Relationship between biodistribution and photodynamic effects in the RIF-1 mouse tumor model. Int. J. Cancer 93, 275-282 http://dx.doi.org/10.1002/ijc.1324

Derycke A. S. L., de Witte P. A. M. (2002): Transferrin-mediated targeting of hypericin embedded in sterically stabilized PEGliposomes. Int. J. Oncol. 20, 181-187

Falk H., Meyer J. (1994): On the homo- and heteroassociation of hypericin. Monatsh. Chem. 125, 753-762 http://dx.doi.org/10.1007/BF01277637

Falk H. (1999): From the photosensitizer hypericin to the photoreceptor stentorin - The chemistry of phenanthroperylene quinones. Angew. Chem. Int. Ed. 38, 3116-3136 http://dx.doi.org/10.1002/(SICI)1521-3773(19991102)38:21< 3116::AID-ANIE3116>3.0.CO;2-S

Fishburn C. S. (2008): The Pharmacology of PEGylation: balancing PD with PK to generate novel therapeutics. J. Pharm. Sci. 97, 4167-4183 http://dx.doi.org/10.1002/jps.21278

Galanou M. C., Theodossiou T. A., Tsiourvas D., Sideratou Z., Paleos C. M. (2008): Interactive transport, subcellular relocation and enhanced phototoxicity of hypericin encapsulated in guanidinylated liposomes via molecular recognition. Photochem. Photobiol. 84, 1073-1083

Gbur P., Dedic R., Chorvat D. Jr., Miskovsky P., Hala J., Jancura D. (2009): Time-resolved luminescence and singlet oxygen formation after illumination of the hypericin-low density lipoprotein complex. Photochem. Photobiol. 85, 816-823 http://dx.doi.org/10.1111/j.1751-1097.2008.00483.x

Hadjur C., Richard M. J., Parat M. A., Jardon P., Favier A. (1996): Photodynamics effects of hypericin on lipid peroxidation and antioxidant status in melanoma cells. Photochem. Photobiol. 64, 375-381 http://dx.doi.org/10.1111/j.1751-1097.1996.tb02474.x

Hamblin M. R., Miller J. L., Rizvi I., Loew H. G., Hasan T. (2003): Pegylation of charged polymer-photosensitizer conjugates: effects of photodynamic efficacy. British J. Cancer 89, 937-943 http://dx.doi.org/10.1038/sj.bjc.6601210

Huntosova V., Alvarez L., Bryndzova L., Nadova Z., Jancura D., Buriankova L., Bonneau S., Brault D., Miskovsky P., Sureau F. (2010): Interaction dynamics of hypericin with low-density lipoproteins and U-87 MG cells. Int. J. Pharmaceutics 389, $32-40$

http://dx.doi.org/10.1016/j.ijpharm.2010.01.010 
Huygens A., Kamuhabwa A. R., de Witte P. A. M. (2005): Stability of different formulations and ion pairs of hypericin. Eur. J. Pharm. Biopharm. 59, 461-468 http://dx.doi.org/10.1016/j.ejpb.2004.09.014

Immordino M. L., Dosio F., Cattel L. (2006): Stealth liposomes: review of the basic science, rationale, and clinical applications, existing and potential. Int. J. Nanomedicine 6, 297-315

Kascakova S., Refregiers M., Jancura D., Sureau F., Maurizot J. C., Miskovsky P. (2005): Fluorescence spectroscopic study of hypericin-photosenzitized oxidation of low-density lipoproteins. Photochem. Photobiol. 81, 1395-1403 http://dx.doi.org/10.1562/2005-04-28-RA-503

Kiesslich T., Krammer B., Plaetzer K. (2006): Cellular mechanisms and prospective applications of hypericin in photodynamic therapy. Curr. Med. Chem. 13, 2189-2204 http://dx.doi.org/10.2174/092986706777935267

Kober M., Pohl K., Efferth T. (2008): Molecular mechanisms underlying St. Johns wort drug interactions. Curr. Drug Metab. 9, 1027-1037 http://dx.doi.org/10.2174/138920008786927767

Kramer B., Verwanger T. (2012): Molecular response to hypericininduced photodamage. Current Med. Chem. 19, 793-798 http://dx.doi.org/10.2174/092986712799034842

Kubin, A., Meissner P., Wierrani F., Burner U., Bodenteich A., Pytel A., Schmeller N. (2008): Fluorescence diagnosis of bladder cancer with new water soluble hypericin bound to polyvinylpyrrolidone: PVP-Hypericin. Photochem. Photobiol. 84, 1560-1563 http://dx.doi.org/10.1111/j.1751-1097.2008.00384.x

Lavie G., Mazur Y., Lavie D., Meruelo D. (1995): The chemical and biological properties of hypericin: a compound with a broad spectrum of biological activities. Med. Res. Rev. 15, 111-119 http://dx.doi.org/10.1002/med.2610150203

Lukyanov A. N., Torchilin V. P. (2004): Micelles from lipid derivatives of water-soluble polymers as delivery systems for poorly soluble drugs. Adv. Drug Del. Rev. 56, 1273-1289 http://dx.doi.org/10.1016/j.addr.2003.12.004

Millard J. W., Alvarex-Nunez F. A., Yalkowsky S. H. (2002): Solubilization by cosolvents-establishing useful constants for the log-linear model. Int. J. Pharm. 245, 153-166 http://dx.doi.org/10.1016/S0378-5173(02)00334-4

Miskovsky P. (2002): Hypericin - a new antiviral and antitumor photosensitizer: Mechanism of action and interaction with biological macromolecules. Curr. Drug Targets 3, 55-84 http://dx.doi.org/10.2174/1389450023348091

Mukherjee P., Adhikary R., Halder M., Petrich J. W., Miskovsky P. (2008): Accumulation and interaction of hypericin in lowdensity lipoproteins - a photophysical study. Photochem. Photobiol. 84, 706-712 http://dx.doi.org/10.1111/j.1751-1097.2007.00234.x

Nandi I., Bateson M., Bari M., Joshi H. N. (2003): Synergistic effect of PEG-400 and cyclodextrin to enhance solubility of progesterone. AAPS PharmSciTech 4, Article 1

Roby A., Erdogan S., Torchilin V. P. (2006): Solubilization of poorly soluble PDT agent, meso-tetraphenylporphin, in plain or immunotargeted PEG-PE micelles results in dramatically improved cancer cell killinig in vitro. Eur. J. Pharm. Biopharm. 62, 235-240 http://dx.doi.org/10.1016/j.ejpb.2005.09.010

Roslaniec M., Weitman H., Freeman D., Mazur Y., Ehrenberg B. J. (2000): Liposome binding constants and singlet oxygen quantum yields of hypericin, tetrahydroxy helianthrone and their derivatives: studies in organic solutions and in liposomes. Photochem. Photobiol. B Biol. 57, 149-158

Saw C. L. L., Olivo M., Chin W. W. L., Soo K. Ch., Heng P. W. S. (2005): Transport of hypericin across chick chorioallantoic membrane and photodynamic therapy vasculature assessment. Biol. Pharm. Bull. 28, 1054-1060 http://dx.doi.org/10.1248/bpb.28.1054

Saw C. L. L., Heng P. W. S, Chin W. W. L., Soo K. C., Olivo M. (2006): Enhanced photodynamic activity of hypericin by penetration enhancer N-methyl pyrrolidone formulations in the chick chorioallontoic membrane model. Cancer Letters 238, 104-110 http://dx.doi.org/10.1016/j.canlet.2005.06.026

Sezgin Z., Yüksel N., Baykara T. (2006): Preparation and charaterization of polymeric micelles for solubilization of poorly soluble anticancer drugs. J. Pharm. Biopharm. 64, 261-268 http://dx.doi.org/10.1016/j.ejpb.2006.06.003

Sezgin Z., Yuksel N., Baykara T. (2007): Investigation of pluronic and PEG-PE micelles as carriers of meso-tetraphenyl porphine for oral administration. Int J. Pharm. 332, 161-167 http://dx.doi.org/10.1016/j.ijpharm.2006.09.030

Torchilin V. P. (2007): Micellar nanocarriers: Pharmaceutical perspectives. Pharm. Res. 24, 1-16 http://dx.doi.org/10.1007/s11095-006-9132-0

van de Putte M., Roskams T., Bormans G., Verbruggen A., de Witte P. A. M. (2006): The impact of aggregation on the biodistribution of hypericin. Int. J. Oncol. 28, 655-660

van Nostrum C. F. (2004): Polymeric micelles to deliver photosensitizers for photodynamic therapy. Adv. Drug Del. 56, 9-16 http://dx.doi.org/10.1016/j.addr.2003.07.013

van Vierken L. E., Vyas T. K., Amiji M. M. (2007): Poly(ethylene glygol)-modified nanocerriers for tumor targeted and intracellular delivery. Phram. Res. 24, 1405-1414

http://dx.doi.org/10.1007/s11095-007-9284-6

Veronese F. M., Pasut G. (2005): PEGylation, successful approach to drug delivery. Drug Discov. Today 21, 1451-1458 http://dx.doi.org/10.1016/S1359-6446(05)03575-0

Wattendorf U., Hans P. M. (2008): PEGylation as a tool for the biomedical engineering of surface modified microparticles. J. Pharm. Sci. 97, 4655-4669 http://dx.doi.org/10.1002/jps.21350

Zeisser-Labouebe M., Lange N., Gurny R., Delie F. (2006): Hypericin-loaded nanoparticles for the photodynamic treatment of ovarian cancer. Int. J. Pharm. 326, 174-181 http://dx.doi.org/10.1016/j.ijpharm.2006.07.012

Zeisser-Labouebe M., Delie F., Gurny R., Lange N. (2009): Benefits of nanoencapsulation for the hypericin-mediated photodetection of ovarian micrometastases. Eur. J. Pharm. Biopharm. 71, $207-213$

http://dx.doi.org/10.1016/j.ejpb.2008.10.005

Received: July 12, 2012

Final version accepted: November 6, 2012 